\title{
Management of Late 3rd Trimester Pregnant in Oral and Maxillofacial Surgery- A Case Report of Submandibular Space Infection
}

\author{
Dr Venkata Ramana Murthy V ${ }^{1^{*}}$, Dr K Chandra Sekhar², Dr T Subbaraju ${ }^{3}$, Dr Vini Kaila ${ }^{4}$, Dr \\ Ganta Gopala Krishna ${ }^{5}$ \\ ${ }^{1}$ M.D.S, Reader, Department of Oral and Maxillofacial Surgery, Vishnu Dental College, Bhimavaram. \\ ${ }^{2}$ M.D Anesthesia, Associate Professor, Department of Anesthesiology, Nri Institute of Medical Sciences, \\ Visakhapatnam \\ ${ }^{3}$ M.D.S, Reader, Sri Sai Dental College and Research Centre, Srikakulam. \\ ${ }^{4}$ M.D.S, Senior Lecturer, Department of Oral and Maxillofacial Surgery, Vishnu Dental College, Bhimavaram \\ ${ }^{5}$ B.D.S, Internee, Vishnu Dental College, Bhimavaram.
}

*Corresponding Author: Dr Venkata Ramana Murthy V, M.D.S, Reader, Deaprtment of Oral and Maxillofacial Surgery, Vishnu Dental College, Bhimavaram Email: murthymaxfac@gmail.com

\begin{abstract}
The purpose of this case report is to emphasize the importance of management of a pregnant patient in the late third trimester \{29th week until child birth\} with huge facial swelling with acute presentation prior to 2 weeks of expected delivery reported to the department of oral and maxillofacial surgery. It's a difficult task to manage a pregnant patient, however in second trimester few elective procedures are carried out but very few cases present with maxillofacial infections in the 3rd trimester, which might increase psychological stress, morbidity to the mother and fetus. The clinicians should be aware of the hemodynamic changes, complications which arise during late stages of pregnancy and appropriate obstetrics consultation which can enhance and promote normalcy towards delivery.
\end{abstract}

Keywords: Third-trimester, Pregnancy, Delivery, Maxillofacial surgery

\section{INTRODUCTION}

Pregnancy in woman is a biological phenomenon which produces many changes in female human body. It is a unique immunological state where natural homeostasis takes place between antigenitically different tissues. Female patients reporting to maxillofacial surgeon during pregnancy require special attention. Maxillofacial infections are commonly odontogenic in origin. Odontogenic infections are self-limiting and confined. They may break through tissue barriers and spread into spaces which may lead to fulminating infections [1].

One should have knowledge to maintain maternal fetal viability and to assure normal pregnancy. Changes observed in pregnant women which suppress the immune system or promote local growth of bacteria which lead to suppressed cellular immunity, altered neutrophil chemotaxis and natural killer cells activity. This might give explanation of multispace maxillofacial infections in pregnant women [2].

\section{CASE REPORT}

A 21 year old female patient reported to the department of oral and maxillofacial surgery with pain, trismus, temperature and progressively enlarging swelling on the right side of the face since 5 days with gross facial asymmetry. On evaluating medical history patient was in $37^{\text {th }}$ week of pregnancy and an expected time of delivery in 2 weeks from the time of presentation and is under iron and folic acid supplementation Dental history revealed that the patient first had pain in the first trimester which was intermittent and without any associated signs and symptoms, later she went to a hospital where it was diagnosed as chronic pulpitis and was treated symptomatically and was advised to undergo root canal therapy in $2^{\text {nd }}$ trimester which the patient has neglected and has not received any definitive treatment in pregnancy. Two days before presentation, she developed acute onset of right side swelling of the cheek associated with pain and discomfort that has progressively exacerabated. 
At the time of presentation, she was febrile, dyspneic, with difficulty in deglutition, phonation, and trismus with severe halitosis. Pus discharge was observed extra orally near the submandibular region with continuous pain from last five days. The patient is a well-nourished in mild distress with increased swelling from last five days.

Her Blood pressure was $140 / 90 \mathrm{~mm}$ of $\mathrm{Hg}$, elevated heart rate of 120 /minute, respirations-20 cycles/minute, Elevated temperature of $39.2^{\circ} \mathrm{C}$, with an oxygen saturation of $96 \%$ on room air was recorded. Significant right-side facial edema measuring $5 \times 4 \mathrm{~cm}$ extending antero-posterioly from tragus to corner of the mouth and from $2 \mathrm{~cm}$ lateral to the corner of eye to $2 \mathrm{~cm}$ inferior border of the mandible superio-inferiorly. The swelling is soft and fluctuant, tender on palpation with pus draining extrarorally with signs of central necrosis. There was tender right submandibular lymphadenopathy.

The patient had difficulty in opening the mouth so intraoral examination was difficult and was advised for orthopantamogram taking care about the exposure to the $\mathrm{x}$-ray which revealed grossly carious 47 with large peri-apical radiolucency and widening of lamina dura diagnosed as submandibular space infection. Treatment was planned after gynecological consultation for extraction of 47, incision and drainage under local anesthesia.

A complete blood count and complete evaluation was done and referral to the obstetrician and gynecology was planned, discussed regarding the precautionary measures to be taken prior to incision and drainage to prevent spread of infection and septicemia, drugs to be used, hypertension control, patient was explained about the consequences motivated and counselled for treatment to undergo incision and drainage with extraction under local anesthesia, antibiotic coverage.

After High Risk Informed Consent, All the necessary precautions were taken priory regarding patient position, hypertension control, and adequate analgesia, planned for a morning appointment, under local anesthesia with lidocaine 2\% with 1:200000 adrenaline. Extraction was performed atraumatically, necrotic skin was excised, incision and drainage was established with corrugated rubber drain and pus was sent for culture sensitivity. Betadine dressings were given regularly for 5 days with cap amoxicillin 500mg bid and metronidazole $100 \mathrm{ml}$ tid IV, injection tramadol 50mg, dietary regular medication as per the gynecologist advise and managed without any postoperative complications. After 2 weeks the patient delivered a baby without any complications.

\section{DISCUSSION}

During pregnancy mother undergoes significant anatomic and physiological changes to nurture and accommodate developing fetus. Various hematologic changes occur with $50 \%$ increase in plasma volume by 34 weeks, fall in hemoglobin concentration, hematocrit, RBC however, there is no change in MCVor MCHC in $3^{\text {rd }}$ trimester [3]. Platelet count at the $3^{\text {rd }}$ trimester falls generally to $100 \times 10^{9}$ cells/L but in this patient $223 \times 10^{9}$ cells/L was seen [4]. There will be requirement of iron with 20fold increase in folate requirements and two folds in $\mathrm{B}_{12}$ Increased risk of venous thrombosis towards third trimester with $50 \%$ increase in fibrinogen levels, decreased fibrinolytic activity, endogenous anticoagulants such as anti-thrombin and protein $\mathrm{S}$ decrease gradually [4].

Blood pressure decreases in $1^{\text {st }}, 2^{\text {nd }}$ trimester but increases to non-pregnant levels in $3^{\text {rd }}$ trimester. Profound effects of maternal position towards term dictates or pose problems so treatment must be done in right or left lateral position as supine position causes $25 \%$ decreased cardiac output [5]. Careful observations for contractions during treatment is a need to avoid unwanted consequences. Hyperventilation, pulmonary edema may develop in cardiovascular compromised women [6].

$40 \%$ fall in renal vasculature, $85 \%$ volume resides in venous circulation realxin hormone stimulates formation of endothelin which inturn mediates vasodilatation of renal arteries. Placental vasodilators are important in maintaining vasodilatory state [7].

The reabsorption of glucose in the proximal and collecting tubules is less effective and $90 \%$ of women will be in normal blood glucose levels tend to excrete $1-10 \mathrm{gms}$ glucose per day. Fractional excretion up to $300 \mathrm{mg} /$ day is also seen. In middle and $3^{\text {rd }}$ trimester fourfold increase in vasopressin is observed [8]. Respiratory inspiratory reserve volume increase in $3^{\text {rd }}$ trimester, decrease in functional residual capacity, and breathlessness without hypoxia is physiological and most common in $3^{\text {rd }}$ trimester [9]. Nausea and vomiting decrease in $3^{\text {rd }}$ trimester. TSH levels rise to $5.5 \mathrm{umol} / \mathrm{L}$, total cortisol levels increase 3 times higher in third trimester. Maternal insulin resistance increases in 
$3^{\text {rd }}$ trimester. LDL cholesterol levels increases to $50 \%$ at term for placental steroidogenesis. HDL levels fall in $3^{\text {rd }}$ trimester [10].

Peak demand for calcium increase in $3^{\text {rd }}$ trimester so increase in dietary absorption from mother has to be enhanced. Bone turnover rate is high in $3^{\text {rd }}$ trimester [11]. All the changes have been clearly monitored with the gynecologist to observe for untoward complications.

\section{CONCLUSION}

In the present day practice of increased risk for medico legal issues, every clinician should be aware of the physiological changes in pregnancy and clinically evaluate the physiology and pathological factors, observe for signs and symptoms and psychologically motivate the mother in diseased state to get treatment done as they deny treatments during pregnancy. It is up to the clinician to take the challenge of managing pregnant women in emergency cases with space infections with active treatment to end up with a safe delivery with a defined clinical and surgical protocol, directed towards optimizing maternal health while minimizing fetal risk.

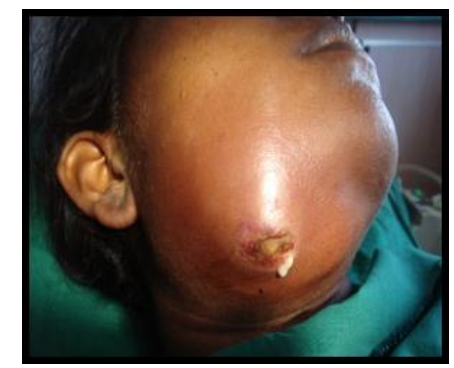

1. Pus Drainage Extraoral

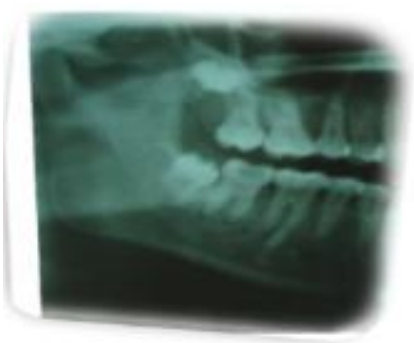

\section{Opg Grossly Decayed 47}

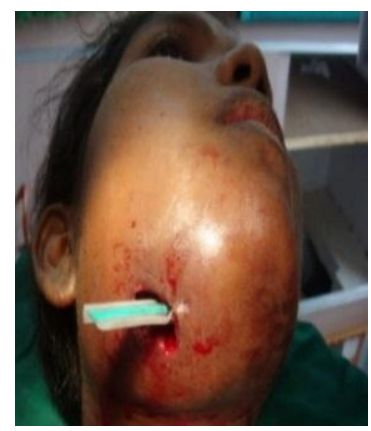

3.Corrugated Drian

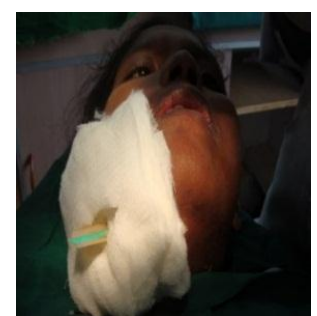

4. Dressing

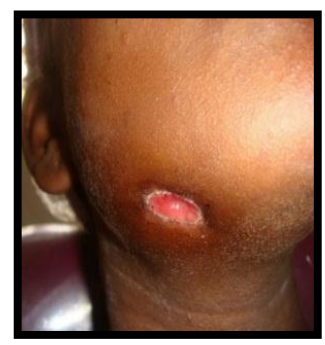

5. Healing Site

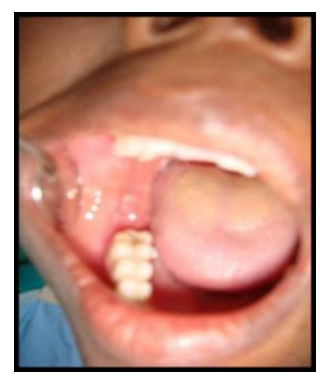

6. Intraoral Socket

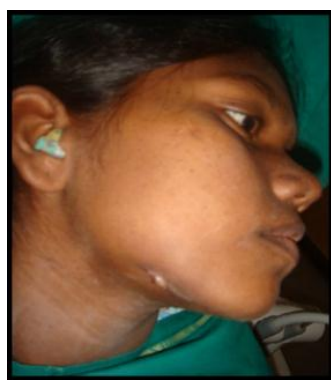

7. Healing Scar

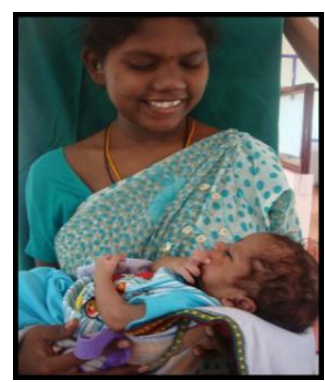

8. Mother and Baby

\section{REFERENCES}

[1] Gill and C. scully: Craniofacial Odontogenic Infection; Review of Microbiology \&Current treatment. Oral surgery, Oral Medicine, Oral Pathology 1990; 70:155-58

[2] Topazian RG; Goldberg M.H; Oral and Maxillofacial infections Philadelphia 1987. 
[3] Clapp JF $3^{\text {rd }}$, Capeless Cardiovascular function before, during, and after the first and subsequent pregnancies.AM J cardiology 1997; 80:1469-73.

[4] Ramsay M.N oral, hematological changes during pregnancy and the puerperium.In Pavord $\mathrm{S}$, Hunt B(ed).The Obstetric Hematology Manual. Cambridge: Cambridge University Press, 2010:3-12.

[5] Gordon MC .Maternal Physiology in Obstetrics: Normal and Problem pregnancies. $6^{\text {th }}$ edn.philadelphia: Saunders, Elsevier, 2012

[6] Wilson M,Morganti AA,Zervoudakis I,Lethcher RL,Romney BM,et al. Blood pressure, the reninaldosterone system and sex steroids throughout normal pregnancy. Am J Med 1980; 68(1):97104
[7] Davidson JM. Renal hemodynamics and volume homeostasis in pregnancy.Scand J Clin Lab Invest Suppl 1984; 169:15-27

[8] Cheung KL, Lafayette RA.Renal Physiology of Pregnancy. Adv Chronic Kidney Dis 2013; 20(3):209-214.

[9] Lee W. cardiorespiratory alterations during normal pregnancy.Crit Care Clin.1991; 7(4):763-75

[10] Weiss G .Endocrinology of parturition. J Clin Endocrinal Metab 2000; 85(12):4421-5

[11] Hamaoui E, Hamaoui M.Nutritional assessment and support during pregnancy. Gastroenterol Clin North Am 2003; 32:59-121

Citation: Dr Venkata Ramana Murthy V, Dr K Chandra Sekhar, Dr T Subbaraju, Dr Vini Kaila, Dr Ganta Gopala Krishna. Management of Late 3rd Trimester Pregnant in Oral and Maxillofacial Surgery-A Case Report of Submandibular Space Infection. ARC Journal of Dental science. 2018; 3(1): 11-14. doi:dx.doi.org/ 10.20431/2456-0030. 0301004 .

Copyright: (C) 2018 Authors. This is an open-access article distributed under the terms of the Creative Commons Attribution License, which permits unrestricted use, distribution, and reproduction in any medium, provided the original author and source are credited. 